\title{
Constraining Higgs boson effective couplings at electron-positron colliders
}

\author{
Hamzeh Khanpour ${ }^{1,2, *}$ and Mojtaba Mohammadi Najafabadi ${ }^{2, \dagger}$ \\ ${ }^{1}$ Department of Physics, University of Science and Technology of Mazandaran, \\ P.O. Box 48518-78195 Behshahr, Iran \\ ${ }^{2}$ School of Particles and Accelerators, Institute for Research in Fundamental Sciences (IPM), \\ P.O. Box 19395-5531 Tehran, Iran
}

(Received 28 September 2016; published 28 March 2017)

\begin{abstract}
We probe the dimension-six operators contributing to Higgs production in association with a $Z$ boson at the future high-luminosity electron-positron colliders. Potential constraints on dimension-six operators in the Higgs sector are determined by performing a shape analysis on the differential angular distribution of the Higgs and $Z$ boson decay products. The analysis is performed at the center-of-mass energies of 350 and $500 \mathrm{GeV}$ including a realistic detector simulation and the main sources of background processes. The $68 \%$ and $95 \%$ confidence level upper limits are obtained on the contributing anomalous couplings considering only the decay of the Higgs boson into a pair of b-quarks and leptonic $Z$ boson decay. Our results show that angular observables provide a great sensitivity to the anomalous couplings, in particular, at the high-luminosity regime.
\end{abstract}

DOI: 10.1103/PhysRevD.95.055026

\section{INTRODUCTION}

Since the Higgs boson discovery at the Large Hadron Collider (LHC) run I in 2012 [1,2], the main task has been to provide precise measurement of its couplings to the standard model (SM) particles as well as its other properties. This opens a way to look for potential new physics effects and provides the possibility for revealing effects that may show up at high energy scales. The recent results of the ATLAS and CMS experiments in probing the couplings of the Higgs boson show no signs of new physics [3]. The Higgs couplings to the SM particles also have been studied extensively in several analyses using available data from the LHC and previous experiments [4-15].

The compatibility of the current measurements with the SM predictions in the Higgs sector causes the new physics scale to be different from the electroweak scale. This suggests searching for new physics effects beyond the SM by adopting the effective field theory (EFT) approach without going through the details of any specific scenarios. In this approach, the effective operators consist of only the SM fields and are obtained by integrating out heavy degrees of freedom. These effective interactions are suppressed by inverse powers of the new physics scale. Such an effective Lagrangian is required to respect the Lorentz symmetry and the $\mathrm{SU}(3)_{\mathrm{C}} \times \mathrm{SU}(2)_{\mathrm{L}} \times \mathrm{U}(1)_{\mathrm{Y}} \mathrm{SM}$ gauge symmetries. Assuming baryon and lepton number conservation, operators of dimension six are the first corrections

\footnotetext{
*Hamzeh.Khanpour@mail.ipm.ir

†Mojtaba@cern.ch
}

that are added to the SM action. The effective Lagrangian can be written as follows:

$$
\mathcal{L}_{\text {eff }}=\mathcal{L}_{\mathrm{SM}}+\sum_{i} \frac{c_{i} \mathcal{O}_{i}}{\Lambda^{2}}
$$

where the effects of possible new physics is assumed to appear at an energy scale of $\Lambda, c_{i}$ coefficients are dimensionless Wilson coefficients, and $\mathcal{O}_{i}$ are dimensionsix operators obtained by integrating out the heavy degrees of freedom in the underlying theory.

So far, there are many studies to constrain these Wilson coefficients in the Higgs boson sector from the LHC run I data and from the electroweak precision tests at large electron-positron (LEP) and future colliders [5-7,16-41]. If the LHC at run II does not observe any significant deviation from the SM expectations, stronger bounds on the coefficients of the effective operators would be set. Realistic estimations of constraints on the effective coefficients of Higgs boson related operators after the LHC run II with high integrated luminosity have been provided in [42].

Electron-positron colliders such as Compact Linear Collider (CLIC) [43-45], International Linear Collider [46-50], Circular Electron-Positron Collider [51,52] or high-luminosity high-precision FCCee [53-62], with clean experimental environment due to the absence of hadronic initial state and accurately known collision energy provide a good opportunity to probe precisely the Higgs boson couplings as well as the measurement of the SM parameters with high accuracy. Going up to high energies and luminosities, these colliders can continue the studies made by LEP and provide an 
excellent place in search for new physics beyond the SM [46,48-50,53,63-70].

In this work, by adopting the effective Lagrangian approach in the strongly interacting light Higgs (SILH) basis $[71,72],{ }^{1}$ we constrain coefficients of dimension-six operators using the Higgs production in association with a $Z$ boson at the electron-positron colliders with the centerof-mass energies of 350 and $500 \mathrm{GeV}$. In Higgs production in association with a $Z$ boson, the correction coming from dimension-six operators is scaled as $s / \Lambda^{2}$ where $s$ is the center-of-mass energy of the collisions and must be greater than $\left(m_{Z}+m_{H}\right)^{2}$ to produce $H+Z$ on shell.

The results are obtained using a realistic simulation including the main background contributions for the $e^{+} e^{-} \rightarrow H+Z$ process. The analysis is based on the channel in which the Higgs boson decays into a pair of b-quarks and $Z$ boson decays leptonically. The upper limits on the coefficients of dimension-six operators are obtained at $68 \%$ and $95 \%$ confidence level using a $\chi^{2}$ analysis on the angular distribution of the Higgs and $Z$ boson decay products. The results are presented for the integrated luminosities of $300 \mathrm{fb}^{-1}$ and $3 \mathrm{ab}^{-1}$.

The present paper is organized as follows: In Sec. II, a brief description of the theoretical framework and assumptions are given. Details of event generation, detector simulation, event selection, and the strategy of the analysis are illustrated in Sec. III. The statistical method used to obtain upper limits on the coefficients of dimension-six operators is presented in Sec. IV. Our results for integrated luminosities of $300 \mathrm{fb}^{-1}$ and $3 \mathrm{ab}^{-1}$ are discussed in Sec. V. Finally, summary and conclusions are given in Sec. VI.

\section{THEORETICAL FRAMEWORK AND ASSUMPTIONS}

In this section, the most general effective Lagrangian up to dimension six containing the SM fields, which respects the gauge and global symmetries of the SM, is introduced. There are equivalent ways to write this effective Lagrangian which cause one to have different bases. In this work, the convention for EFT operators proposed in Refs. [42,71,72] is followed. Considering baryon and lepton number conservation, the relevant parts of the effective Lagrangian that affect the Higgs boson couplings have the following terms:

$$
\mathcal{L}_{\mathrm{EFT}}=\mathcal{L}_{\mathrm{SM}}+\mathcal{L}_{\mathrm{SILH}}+\mathcal{L}_{\mathrm{F}_{1}}+\mathcal{L}_{\mathrm{F}_{2}},
$$

where $\mathcal{L}_{\text {SILH }}$ consists of a set of $C P$-even dimension-six operators involving the Higgs doublet and is inspired from models in which the Higgs field is part of a strongly interacting sector [73]. The third term, $\mathcal{L}_{\mathrm{F}_{1}}$, contains interactions among two Higgs fields and a pair of leptons or quarks. The fourth term of the effective Lagrangian, $\mathcal{L}_{\mathrm{F}_{2}}$, expresses the interactions of a quark or lepton pair with a single Higgs field and a gauge boson. For instance, $\mathcal{L}_{\text {SILH }}$ has the following form:

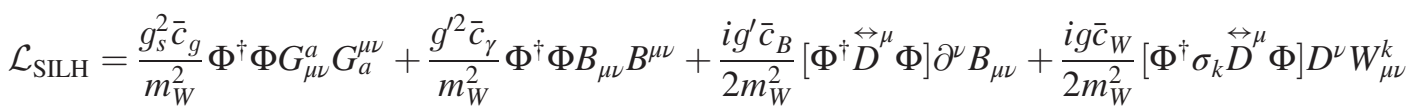

$$
\begin{aligned}
& +\frac{i g \bar{c}_{H W}}{m_{W}^{2}}\left[D^{\mu} \Phi^{\dagger} \sigma_{k} D^{\nu} \Phi\right] W_{\mu \nu}^{k}+\frac{i g^{\prime} \bar{c}_{H B}}{m_{W}^{2}}\left[D^{\mu} \Phi^{\dagger} D^{\nu} \Phi\right] B_{\mu \nu}+\frac{\bar{c}_{H}}{2 v^{2}} \partial^{\mu}\left[\Phi^{\dagger} \Phi\right] \partial_{\mu}\left[\Phi^{\dagger} \Phi\right]+\frac{\bar{c}_{T}}{2 v^{2}}\left[\Phi^{\dagger} \stackrel{\leftrightarrow}{D} \mu \mid \Phi\right]\left[\Phi^{\dagger} \stackrel{\leftrightarrow}{D}_{\mu} \Phi\right] \\
& -\frac{\bar{c}_{6} \lambda}{v^{2}}\left[\Phi^{\dagger} \Phi\right]^{3}-\left[\frac{\bar{c}_{l}}{v^{2}} y_{\ell} \Phi^{\dagger} \Phi \Phi \bar{L}_{L} e_{R}+\frac{\bar{c}_{u}}{v^{2}} y_{u} \Phi^{\dagger} \Phi \Phi^{\dagger} \cdot \bar{Q}_{L} u_{R}+\frac{\bar{c}_{d}}{v^{2}} y_{d} \Phi^{\dagger} \Phi \Phi \bar{Q}_{L} d_{R}+\text { H.c. }\right] \text {, }
\end{aligned}
$$

where $\Phi$ is a weak doublet that contains the Higgs boson field and $B^{\mu \nu}, W^{\mu \nu}, G^{\mu \nu}$ are the electroweak and strong field strength tensors. The Hermitian covariant derivative is defined as $\Phi^{\dagger} \stackrel{\leftrightarrow}{D} \varphi=\Phi^{\dagger}\left(D^{\mu} \Phi\right)-\left(D^{\mu} \Phi\right)^{\dagger} \Phi$. The Higgs quartic coupling is denoted by $\lambda$ and $v$ is the weak scale that is defined as $v=1 /\left(\sqrt{2} G_{F}\right)^{1 / 2}=246 \mathrm{GeV} . \bar{c}_{u}, \bar{c}_{d}$, and $\bar{c}_{l}$ are real parameters as the Higgs boson is assumed to be a $C P$-even particle.

Accuracy of the oblique parameters $S$ and $T$ from the electroweak precision measurements leads us to reduce the number of parameters in the above effective Lagrangian. The per-mille constraints on $S$ and $T$ parameters lead to $\bar{c}_{T}=0$ and $\bar{c}_{B}+\bar{c}_{W}=0$ as these are directly related to the oblique parameters [73-76].

\footnotetext{
${ }^{1}$ This basis is not unique and could be connected to other bases.
}

The effective Lagrangian describing the Higgs boson couplings has been studied at CLIC with $1 \mathrm{ab}^{-1}$ of integrated luminosity at the center-of-mass energy of $3 \mathrm{TeV}$ [50]. The study has been performed through double Higgs production as the vertices involving more than a Higgs boson can provide the possibility for testing the composite nature of the Higgs boson. The sensitivity reach has been reported in the plane of $\xi$ and $m_{\rho}$ where $m_{\rho}$ is the mass scale of the heavy strong sector resonances and $\xi=\frac{v}{f}$. $f$ is the compositeness scale and $v$ is the vacuum expectation value. A detailed description of these parameters can be found in [73]. According to this study, the region of $\xi>0.03$ could be excluded at $95 \%$ confidence level (CL) for any value of $m_{\rho}$.

In this analysis, we consider the effects of $\mathcal{L}_{\text {EFT }}$ [Eq. (2)] in the $e^{-}+e^{+} \rightarrow H+Z$ process and the contributions from any other possible effective operators are neglected. 


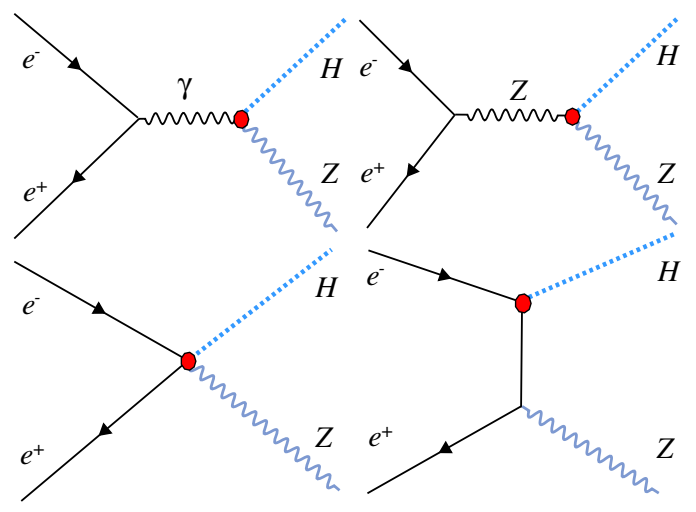

FIG. 1. Representative tree level Feynman diagrams for the production of a Higgs boson in association with a $Z$ boson at an electron-positron collider in the presence of dimension-six operators.

The SM tree level part contribution is not dependent on the momenta of the particles, while $\mathcal{L}_{\mathrm{EFT}}$ introduces momentum-dependent interactions. As a result, the new contributions from $\mathcal{L}_{\mathrm{EFT}}$ affect the decay rates, production cross sections, as well as the shape of differential distributions. In this paper, by exploiting differences in the shape of angular distributions of the decay products of the Higgs and $Z$ bosons, the new involved couplings from $\mathcal{L}_{\mathrm{EFT}}$ in $e^{-}+e^{+} \rightarrow H+Z$ process are studied. The representative Feynman diagrams for production of a Higgs boson in association with a $Z$ boson are depicted in Fig. 1. The vertices affected by $\mathcal{L}_{\mathrm{EFT}}$ are presented by filled circles.

The $e^{-}+e^{+} \rightarrow H+Z$ process is sensitive to the following set of $\mathcal{L}_{\mathrm{EFT}}$ parameters:

$$
\bar{c}_{\gamma}, \bar{c}_{H W}, \bar{c}_{H B}, \bar{c}_{W}, \bar{c}_{B}, \bar{c}_{H}, \bar{c}_{T}, \bar{c}_{e W}, \bar{c}_{e B}, \bar{c}_{l} \text {. }
$$

The parameters $\bar{c}_{e W}$ and $\bar{c}_{e B}$ are coming from $\mathcal{L}_{\mathrm{F}_{2}}$ in Eq. (2) where the related terms contain electron Yukawa coupling $y_{e}$. As we mentioned before, the precise measurement of oblique parameters $S$ and $T$ leads $\bar{c}_{T}=0$ and $\bar{c}_{W}=-\bar{c}_{B}$, which reduces the number of degrees of freedom from 10 to 8 . Because of very small Yukawa coupling of the electron, $\bar{c}_{l}, \bar{c}_{e W}$, and $\bar{c}_{e B}$ do not lead to considerable modifications in the cross section. Consequently, we limit ourselves to only the remaining five parameters: $\bar{c}_{\gamma}, \bar{c}_{H W}$, $\bar{c}_{H B}, \bar{c}_{W}, \bar{c}_{H}$.

Another approach to present the effective Lagrangian that is interesting phenomenologically and experimentally is the effective Lagrangian in the mass basis. This approach has been found to be a useful approach for electroweak precision tests. Following Ref. [71], the relevant subset of the anomalous Higgs boson couplings in the mass basis includes

$$
\begin{aligned}
\mathcal{L}= & -\frac{1}{4} g_{h z z}^{(1)} Z_{\mu \nu} Z^{\mu \nu} h-g_{h z z}^{(2)} Z_{\nu} \partial_{\mu} Z^{\mu \nu} h+\frac{1}{2} g_{h z z}^{(3)} Z_{\mu} Z^{\mu} h \\
& -\frac{1}{2} g_{h a z}^{(1)} Z_{\mu \nu} F^{\mu \nu} h-g_{h a z}^{(2)} Z_{\nu} \partial_{\mu} F^{\mu \nu} h,
\end{aligned}
$$

where the relation between the couplings in the mass basis and the dimension-six coefficients are given as below,

$g_{h z z}^{(1)}=\frac{2 g}{c_{W}^{2} m_{W}}\left[\bar{c}_{H B} s_{W}^{2}-4 \bar{c}_{\gamma} s_{W}^{4}+c_{W}^{2} \bar{c}_{H W}\right]$,

$\left.g_{h z z}^{(2)}=\frac{g}{c_{W}^{2} m_{W}}\left[\left(\bar{c}_{H W}+\bar{c}_{W}\right) c_{W}^{2}+\left(\bar{c}_{B}+\bar{c}_{H B}\right) s_{W}^{2}\right)\right]$,

$g_{h z z}^{(3)}=\frac{g m_{W}}{c_{W}^{2}}\left[1-\frac{1}{2} \bar{c}_{H}-2 \bar{c}_{T}+8 \bar{c}_{\gamma} \frac{s_{W}^{4}}{c_{W}^{2}}\right]$,

$g_{\text {haz }}^{(1)}=\frac{g s_{W}}{c_{W} m_{W}}\left[\bar{c}_{H W}-\bar{c}_{H B}+8 \bar{c}_{\gamma} s_{W}^{2}\right]$,

$g_{\text {haz }}^{(2)}=\frac{g s_{W}}{c_{W} m_{W}}\left[\bar{c}_{H W}-\bar{c}_{H B}-\bar{c}_{B}+\bar{c}_{W}\right]$.

Detailed information together with a complete list of anomalous couplings of the Higgs boson in the mass basis can be found in [71].

We calculate the effects of the dimension-six operators on $H+Z$ production with Monte Carlo (MC) simulations using MadGraph5-aMC@NLO [77-79]. The Lagrangian introduced in Eq. (2) has been implemented in the FeynRule package [80] and then in MadGraph5aMC@NLO, which can be found in Refs. [71,72]. In the next sections, the details of simulation and determination of the $68 \%$ and $95 \%$ CL limits on the coefficients of dimensionsix operators are described.

\section{SIMULATION DETAILS AND ANALYSIS}

In this section, the details of simulation for probing the effective Lagrangian through the $H+Z$ events in the electron-positron collisions are discussed. We focus on the Higgs decay into a pair of b-quarks and $Z$ boson decay into a pair of charged leptons, $(\ell=e, \mu)$. As a result, the final state consists of two energetic jets originating from the hadronization of two b-quarks as well as two charged leptons. The dominant background processes that are considered in this analysis are (i) $e^{+} e^{-} \rightarrow Z Z$ in which one $Z$ decays hadronically and another one decays into charged leptons; (ii) $e^{+} e^{-} \rightarrow t \bar{t}$ in the dilepton final state, which contains two b-jets, two charged leptons, and missing energy; (iii) $e^{+} e^{-} \rightarrow Z \gamma \rightarrow \ell^{+} \ell^{-} j j$ and $e^{+} e^{-} \rightarrow \gamma \gamma \rightarrow \ell^{+} \ell^{-} j j$; and (iiii) $e^{+} e^{-} \rightarrow W^{+} W^{-} Z$ either with leptonic decay of both $W$ bosons and hadronic decay of the $Z$ boson or with hadronic decay of the $W$ bosons and leptonic decay of the $Z$ boson.

Signal and background processes are generated with MadGraph5-aMC@NLO [77-79] event generator and are passed through PYTHIA 8 [81,82] for parton showering, hadronization, and decay of unstable particles. Delphes $3.3 .2[83,84]$ is employed to account for the detector effects similar to an International Linear Detector (ILD)-like detector [47]. The SM input parameters are taken as the following [85]: $m_{H}=125.0 \mathrm{GeV}, m_{t}=173.34 \mathrm{GeV}$, $m_{W}=80.385 \mathrm{GeV}$, and $m_{Z}=91.187 \mathrm{GeV}$. 
The tracking efficiency of an ILD-like detector is set to $99 \%$ for charged particles with $p_{T}>0.1 \mathrm{GeV}$ and $|\eta| \leq 2.4$, including electrons and muons. Electrons, muons, and photons with transverse momenta greater that $10 \mathrm{GeV}$ are reconstructed with an efficiency of $99 \%$
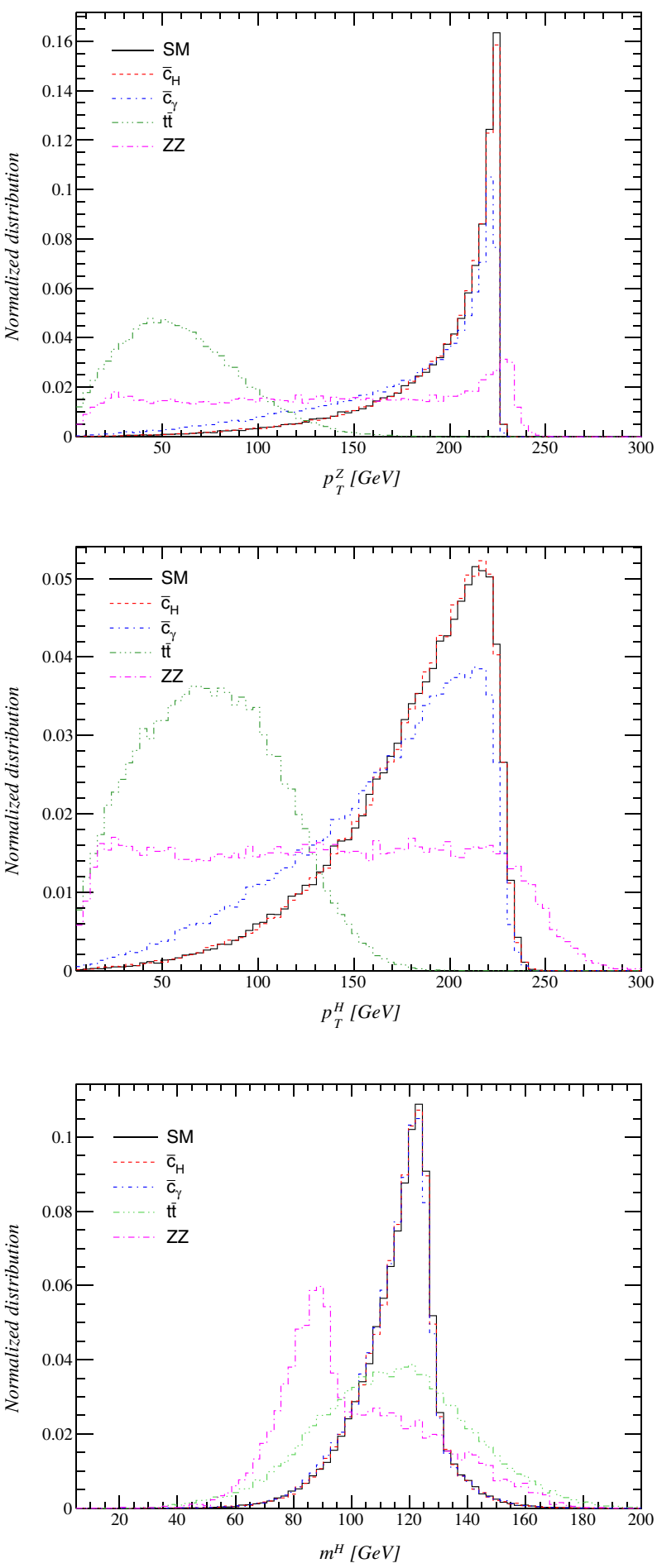

in an ILD-like detector. The momentum resolution for muons is $\Delta p / p=\left(1.0+0.01 \times p_{T}[\mathrm{GeV}]\right) \times 10^{-3}$ for $|\eta| \leq 1 \quad$ and $\quad \Delta p / p=\left(1.0+0.01 \times p_{T}[\mathrm{GeV}]\right) \times 10^{-2}$ for $1<|\eta| \leq 2.4$. The electron and jet energy resolutions are assumed to be
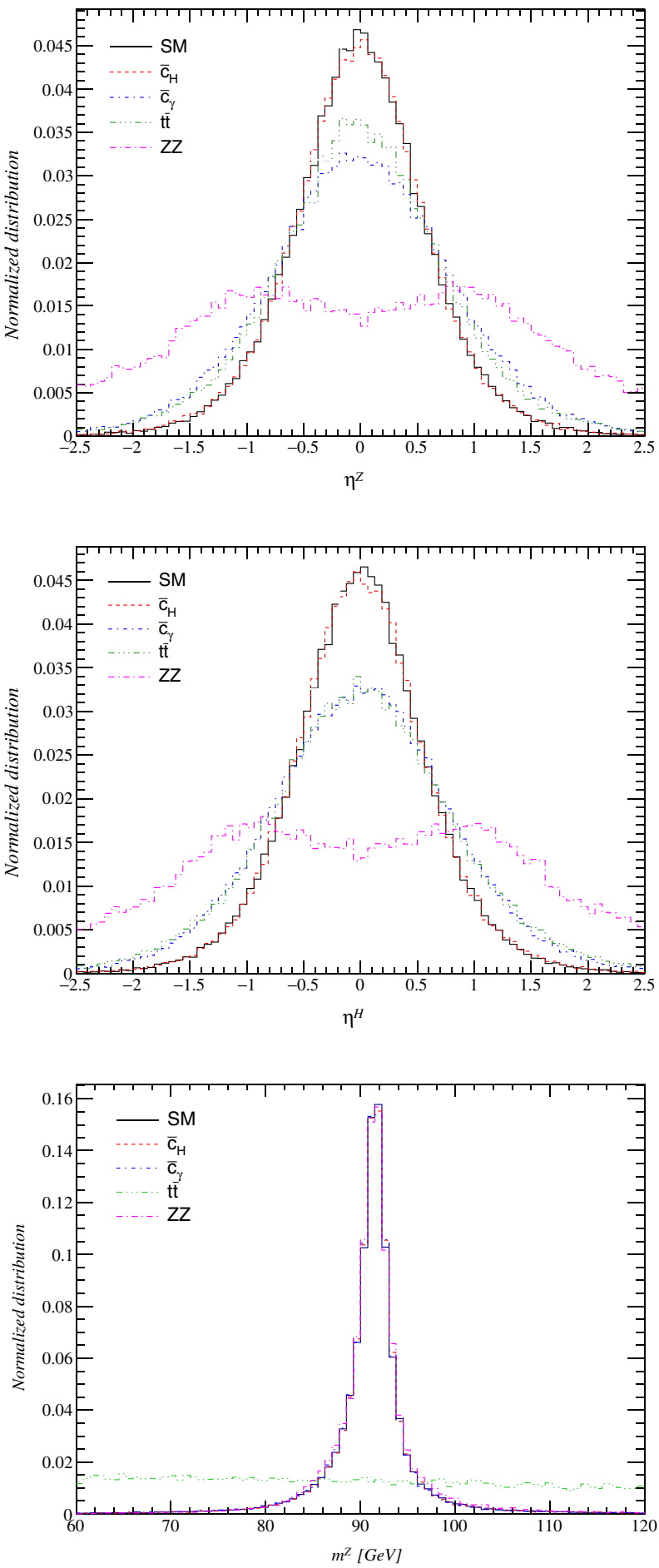

FIG. 2. The transverse momentum and pseudorapidity, and mass distributions of the reconstructed $Z\left(\ell^{+} \ell^{-}\right)$and Higgs boson $(b \bar{b})$ for particular values of $\bar{c}_{H}=0.1, \bar{c}_{\gamma}=0.1$ and SM background processes. The distributions are depicted after the preselection cuts. 
TABLE I. Expected cross sections in units of fb after different combinations of cuts for signal and SM background processes. The signal cross sections are corresponding to particular values of $\bar{c}_{H}=0.1$ and $\bar{c}_{\gamma}=0.1$. The center-of-mass energy of the collision is assumed to be $500 \mathrm{GeV}$. More details of the selection cuts are given in the text.

\begin{tabular}{|c|c|c|c|c|c|c|}
\hline \multirow{2}{*}{$\begin{array}{l}\sqrt{s}=500 \mathrm{GeV} \\
\text { Cuts }\end{array}$} & \multicolumn{2}{|c|}{ Signal } & \multicolumn{4}{|c|}{ Background } \\
\hline & $\bar{c}_{H}$ & $\bar{c}_{\gamma}$ & $\mathrm{SM}(H+Z)$ & $t \bar{t}$ & $Z Z$ & $Z \gamma, \gamma \gamma, W W Z$ \\
\hline Cross sections (in fb) & 4.51 & 16.76 & 5.00 & 24.77 & 36.16 & 11.47 \\
\hline (I): $2 \ell,\left|\eta^{\ell}\right|<2.5, p_{T}^{\ell}>10$ & 3.41 & 12.22 & 3.79 & 15.18 & 23.27 & 7.37 \\
\hline (II): 2 jets, $\left|\eta^{\text {jet }}\right|<2.5, p_{T}^{\text {jet }}>20, \Delta R_{\ell \text {,jet }} \geq 0.5$ & 2.48 & 8.81 & 2.75 & 11.21 & 13.95 & 4.52 \\
\hline (III): $2 b$-jets & 1.09 & 3.84 & 1.22 & 4.71 & 1.16 & 0.35 \\
\hline (IV): $p_{T}^{\ell^{+} \ell^{-}}>100$ & 1.06 & 3.56 & 1.18 & 0.51 & 0.73 & 0.094 \\
\hline (V): $90<m_{b \bar{b}}<160,75<m_{\ell^{+} \ell^{-}}<105$ & 0.921 & 3.040 & 1.022 & 0.078 & 0.138 & 0.003 \\
\hline
\end{tabular}

$$
\begin{aligned}
\frac{\Delta E_{\text {electron }}}{E_{\text {electron }}} & =\frac{15 \%}{\sqrt{E_{\text {electron }}(\mathrm{GeV})}}+1.0 \%, \\
\frac{\Delta E_{\text {jets }}}{E_{\text {jets }}} & =\frac{50 \%}{\sqrt{E_{\text {jets }}(\mathrm{GeV})}}+1.5 \% .
\end{aligned}
$$

Jets are reconstructed with the anti- $k_{t}$ algorithm [86] using the Fast Jet package [87] with a cone size parameter $\mathrm{R}=0.5$. The b-tagging efficiency and misidentification rates depend on the jet transverse momentum and are taken according to an ILD-like detector [47]. At a transverse momentum of around $50 \mathrm{GeV}$, the b-tagging efficiency is around $64 \%$, c-jet misidentification rate is $17 \%$, and a misidentification rate of the light jet is around $1.2 \%$.

We select the signal and background events according to the following requirements: Exactly two same flavor opposite sign charged leptons $(\ell=e, \mu)$ with the transverse momentum $p_{T}^{\ell}>10 \mathrm{GeV}$ and the pseudo-rapidity of $\left|\eta_{\ell}\right| \leq$ 2.5 are required. Each event is required to have only two b-tagged jets with $p_{T}^{\text {jets }}>20 \mathrm{GeV}$ and $\left|\eta_{\text {jets }}\right| \leq 2.5$. To make sure all objects are well isolated, we require that the angular separation $\Delta R_{\ell, \text { b-jets }}=\sqrt{(\Delta \phi)^{2}+(\Delta \eta)^{2}}>0.5$. The above cuts are denoted as the preselection cuts.

To reduce the contributions from all background processes without a Higgs boson or a $Z$ boson in the final state, window cuts on the reconstructed Higgs boson and $Z$ boson are applied. It is required that $90<m_{b \bar{b}}<160 \mathrm{GeV}$ and $75<m_{\ell \ell}<105 \mathrm{GeV}$. In Figs. 2, we show the transverse momentum, pseudorapidity, and mass distributions of the reconstructed Higgs ( $b \bar{b}$-pair) and $Z\left(l^{+} l^{-}\right.$-pair) bosons for center-of-mass energy of $\sqrt{s}=500 \mathrm{GeV}$ for the SM background processes and for the signal processes with $\bar{c}_{H}=0.1$ and $\bar{c}_{\gamma}=0.1$. The distributions are depicted after the preselection cuts. As it can be seen, the reconstructed Higgs and $Z$ bosons in signal events tend to reside at high transverse momentum region while the $t \bar{t}$ background process is in low transverse momentum region. As a result, the transverse momentum distributions of Higgs or $Z$ bosons are good variables to suppress the contribution of the $t \bar{t}$ background process. In addition to the above selection, an additional cut on the $Z$ boson transverse momentum is applied. Because of the correlation between the transverse momenta of the $Z$ boson and Higgs boson, only the cut is applied on one of them.
Cross sections of signal and background processes after imposing each set of cuts are presented in Table I. According to Table I, the cut on transverse momentum of the reconstructed $Z$ boson $\left(p_{T}^{\ell^{+} \ell^{-}}\right)$and the window cuts on the reconstructed Higgs and $Z$ boson masses efficiently reject the background contributions and keep the signal events. In particular, these cuts are very useful to reduce the $t \bar{t}$ background process and $\gamma \gamma, Z \gamma, W^{+} W^{-} Z$ backgrounds.

In order to achieve good sensitivity to the new effective couplings and find the exclusion regions for $c_{i}$, a shape analysis on an angular distribution of the final state particles for which the shape of signal is different from background processes is performed. Figure 3 shows the distribution of the cosine of the angle between the highest $p_{T}$ b-jet and the highest $p_{T}$ charged lepton, $\cos (\ell, b)$, for the signal and for the SM background processes after the preselection cuts. For the $H+Z$ signal, the charged lepton and $\mathrm{b}$-jet tend to be produced mostly back to back at

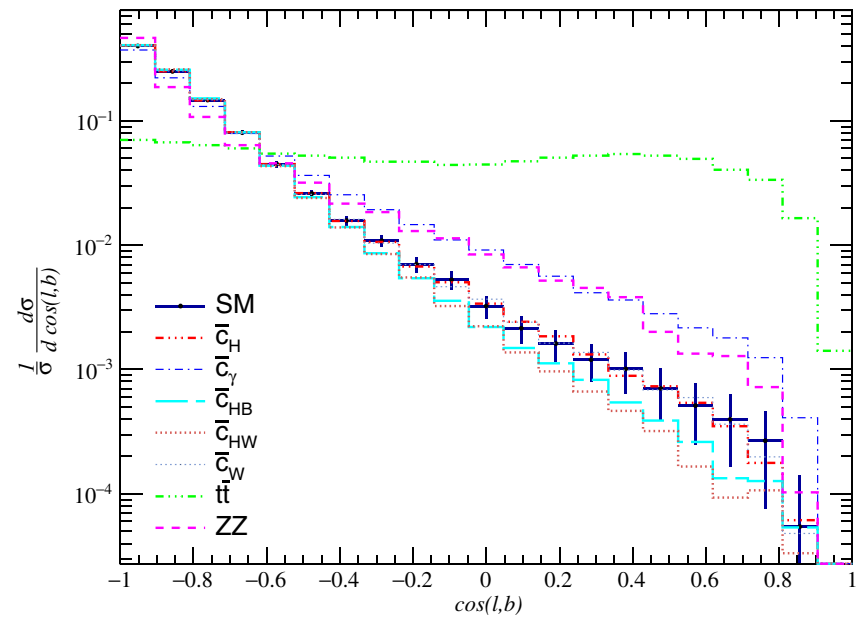

FIG. 3. The $\cos (\ell, b-$ jets $)$ distributions for SM production of the Higgs boson in association with a $Z$ boson and $H+Z$ production in the present various couplings at the center-of-mass energy of $\sqrt{s}=500 \mathrm{GeV}$. The distributions are depicted after the preselection cuts. The signal distributions are presented for particular values of the coupling set to 0.1 . The distributions of two main background processes $t \bar{t}$ and $Z Z$ are depicted for more illustration. The uncertainty on the SM $H+Z$ production is only the statistical uncertainty corresponding to the integrated luminosity of $300 \mathrm{fb}^{-1}$. 
$\cos (\ell, b) \approx-1$. As it can be seen, some of the dimensionsix operators could modify the shape of the $\cos (\ell, b)$ distribution with respect to the SM production of Higgs associated with a $Z$ boson. For example, switching on $\bar{c}_{\gamma}$ leads to an increase of the number of events in the region of $\cos (\ell, b)>0$ while nonzero value of $\bar{c}_{H W}$ leads to a decrease of the number of events in the region of $\cos (\ell, b)>0$ with respect to the SM Higgs production in association with a $Z$ boson. The $t \bar{t}$ background process has almost a flat distribution while the $Z Z$ process has a shape almost similar to the $\bar{c}_{\gamma}$ signal process.

At this point, it should be mentioned that other distributions such as the Higgs boson transverse momentum that differentiates between signal and background processes could be used to derive limits on the new couplings. In particular, performing a simultaneous likelihood fit on both distributions $\left[\cos (\ell, b)\right.$ and $\left.p_{T}^{H}\right]$ would lead to better results. In the present work, only the $\cos (\ell, b)$ distributions of signal and background processes are used to obtain the upper limits on the new effective couplings.

\section{STATISTICAL METHOD}

In order to obtain exclusion regions in the $\left(\bar{c}_{i} ; \bar{c}_{j}\right)$ plane, where $\bar{c}_{i, j}$ are coefficients of the dimension-six operators defined in Eq. (2), a binned $\chi^{2}$ analysis is performed on the $d \sigma / d \cos (\ell, b)$ distribution. At a time, we switch on two effective couplings $\left(\bar{c}_{i} ; \bar{c}_{j}\right)$ as well as the $\mathrm{SM} H+Z$ process and all background processes with the same final state. Therefore, the $\chi^{2}$ is a function of two effective couplings $\left(\bar{c}_{i} ; \bar{c}_{j}\right)$ and has the following form:

$$
\chi^{2}\left(\bar{c}_{i} ; \bar{c}_{j}\right)=\sum_{i}^{n_{\text {bins }}}\left[\frac{N_{i}^{\mathrm{th}}\left(\bar{c}_{i} ; \bar{c}_{j}\right)-N_{i}^{\mathrm{exp}}}{\Delta N_{i}^{\mathrm{exp}}}\right]^{2}
$$

where $N_{i}^{\text {th }}\left(\bar{c}_{i} ; \bar{c}_{j}\right)=\sigma_{i}\left(\bar{c}_{i} ; \bar{c}_{j}\right) \times \epsilon_{i} \times B(H \rightarrow b \bar{b}) \times \mathcal{L}$ and $N_{i}^{\text {exp }}$ are the number of signal and SM expected events in the $i$ th bin of the $\cos (\ell, b)$ distribution. $\sigma_{i}\left(\bar{c}_{i} ; \bar{c}_{j}\right)$ is the cross section of the signal process in the $i$ th bin of the $\cos (\ell, b)$ distribution and $\mathcal{L}$ is the integrated luminosity.
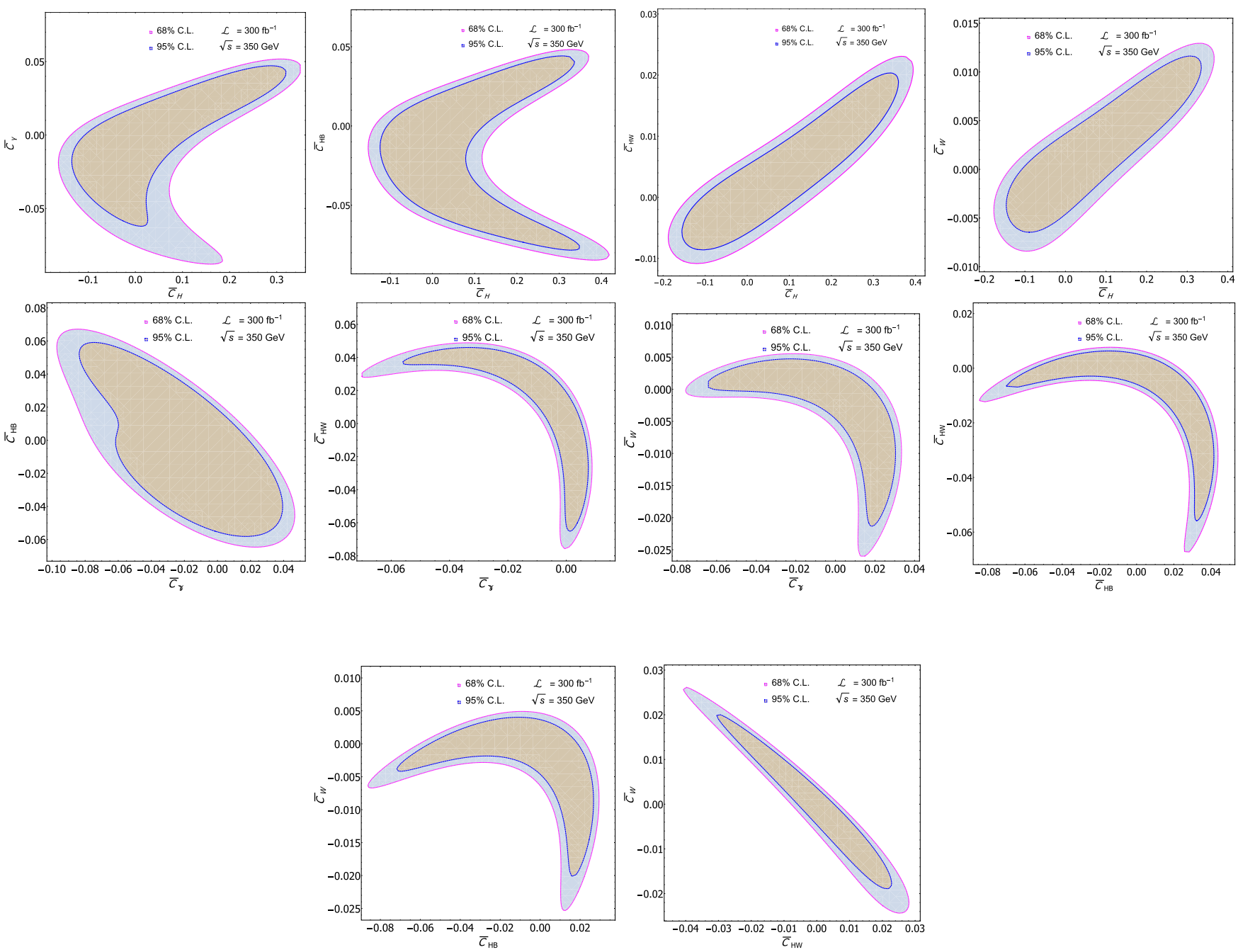

FIG. 4. Contours of $68 \%$ and $95 \%$ confidence level obtained from a fit using the $\cos (\ell, b-$ jets $)$ distributions for $\sqrt{s}=350 \mathrm{GeV}$ with a luminosity of $300 \mathrm{fb}^{-1}$. 
The selection efficiency in each bin is denoted by $\epsilon_{i}$ and $B(H \rightarrow b \bar{b})$ is the branching fraction of Higgs boson decay into the $b \bar{b}$ pair in the SM framework. For $m_{H}=125 \mathrm{GeV}$, the value of the branching fraction of Higgs boson decay into $b \bar{b}$ is 0.584 with the relative theoretical uncertainty of +0.032 and -0.033 [85]. The combined statistical and systematic uncertainties in each bin is denoted by $\Delta N_{i}^{\exp }$. It is defined as $\Delta N_{i}^{\mathrm{exp}}=\sqrt{N_{i}^{\mathrm{SM}+\mathrm{bkg}}\left(1+\Delta_{\text {sys }}^{2} \times N_{i}^{\mathrm{SM}+\mathrm{bkg}}\right)}$ where $\Delta_{\text {sys }}$ reflects the effect of an overall systematic uncertainty. In this work, the results are presented with and without considering any systematic effects. The predicted constraints at $95 \% \mathrm{CL}$ considering only one Wilson coefficient in the above fit are obtained as well.

\section{ANALYSIS RESULTS}

In this section the results of the analysis are presented for the electron-positron collisions at the center-of-mass energies of 350 and $500 \mathrm{GeV}$. The expected two-dimensional contours at $68 \%$ and $95 \%$ confidence level on $\left(c_{i}, c_{j}\right)$ coefficients are shown in Figs. 4 and 5 for the integrated luminosities of $300 \mathrm{fb}^{-1}$ and $3 \mathrm{ab}^{-1}$ of collisions at $\sqrt{s}=350 \mathrm{GeV}$. For comparison, the results are also presented for the center-of-mass energy collision of $500 \mathrm{GeV}$ in Fig. 6. For both integrated luminosities and both energies, one can clearly see that the limits on the coefficients $\left(\bar{c}_{H W}, \bar{c}_{W}\right)$ are considerably stronger than the other coefficients.

At the center-of-mass energy of $350 \mathrm{GeV}$, for some Wilson coefficients, increasing the integrated luminosity from $300 \mathrm{fb}^{-1}$ to $3 \mathrm{ab}^{-1}$ can improve the constraints by a factor of around 2 and some by a factor of 3 .

From Table II, where the bounds from one dimensional fit are extracted, it can be seen that going to higher energy of the electron-positron collisions, from 350 to $500 \mathrm{GeV}$, leads to improvements for the Wilson coefficients. For example, the constraints obtained from $350 \mathrm{GeV}$ with the integrated luminosity of $300 \mathrm{fb}^{-1}$ on $\bar{c}_{W}$ are $-0.00480<\bar{c}_{W}<0.00379$,
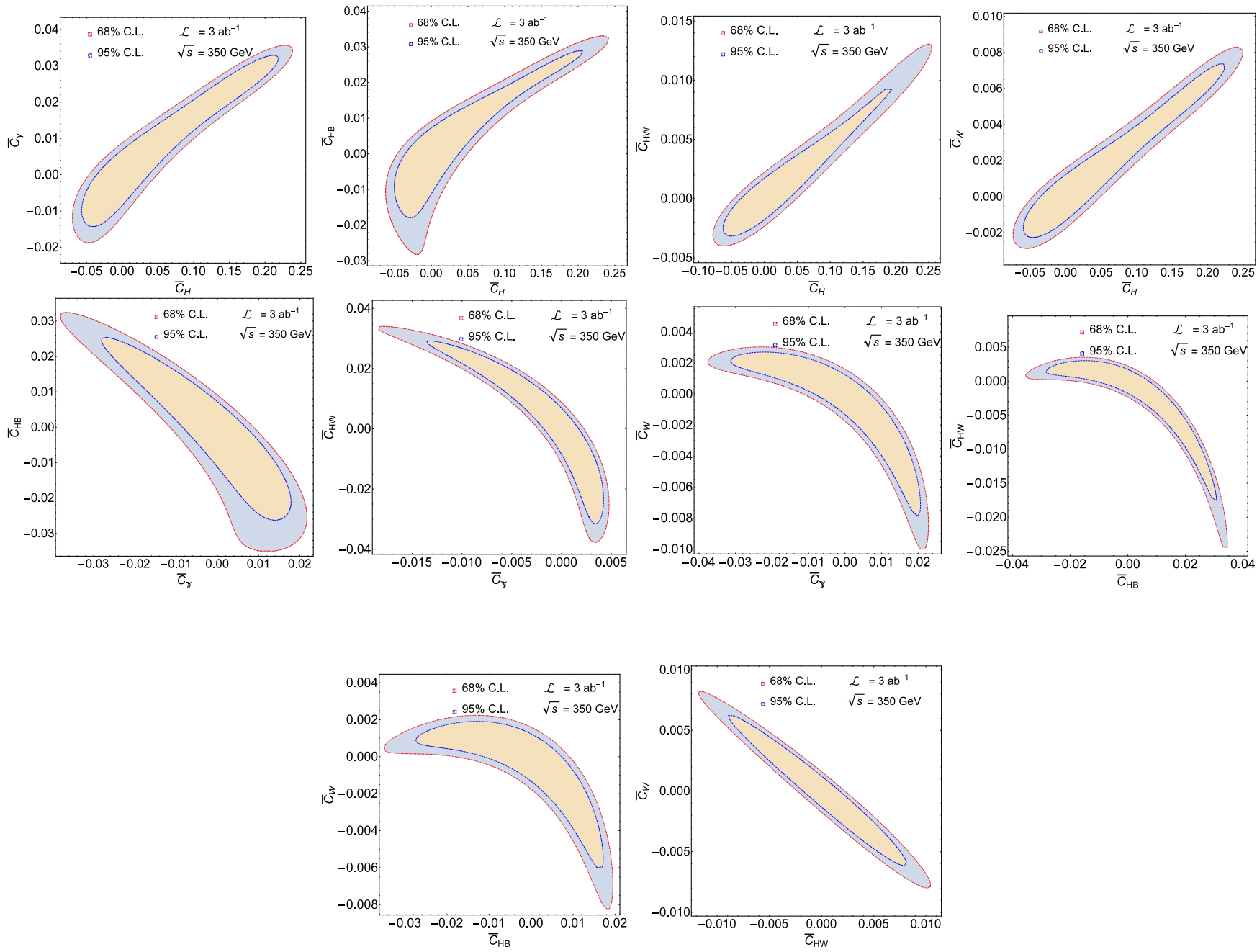

FIG. 5. Contours of $68 \%$ and $95 \%$ confidence level obtained from a fit using the $\cos (\ell, b-$ jets $)$ distributions for $\sqrt{s}=350 \mathrm{GeV}$ with an integrated luminosity of $3 \mathrm{ab}^{-1}$. 

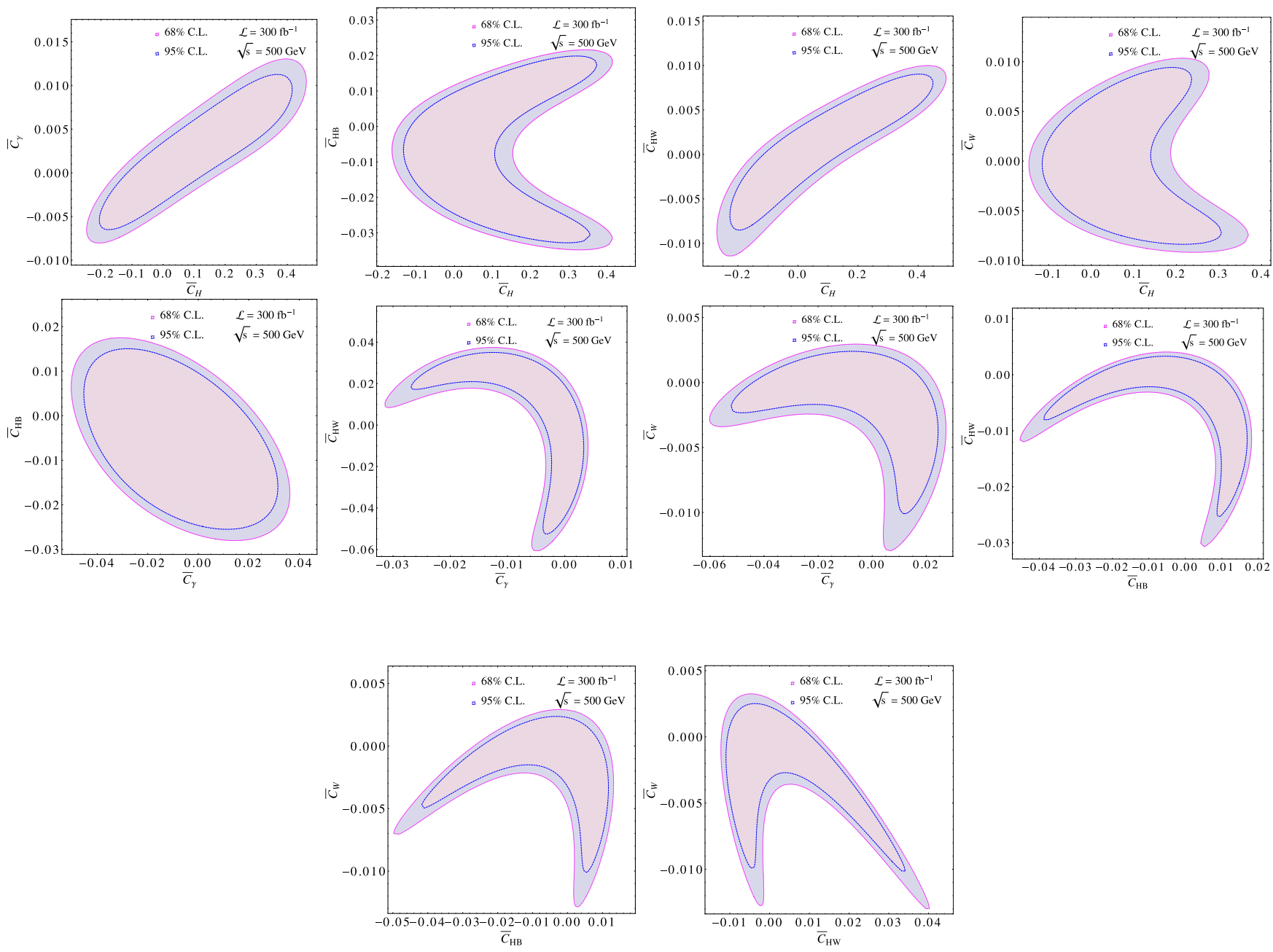

FIG. 6. Contours of $68 \%$ and $95 \%$ confidence level obtained from a fit using the $\cos (\ell, b-$ jets $)$ distributions for the center-of-mass energy of $\sqrt{s}=500 \mathrm{GeV}$ with an integrated luminosity of $300 \mathrm{fb}^{-1}$.

which is tightened as $-0.00324<\bar{c}_{W}<0.00231$ at a $500 \mathrm{GeV}$ machine.

It is instructive to compare the sensitivity of the bounds expected from the high-luminosity LHC with the bounds obtained here in this study. In Table II, the results of this analysis are compared the ones expected to be achieved by the LHC with the integrated luminosities of $300 \mathrm{fb}^{-1}$ and $3 \mathrm{ab}^{-1}$ for the case of considering only one Wilson coefficient in the fit. In [42], the constraints on the Wilson coefficients have been obtained at the LHC at $14 \mathrm{TeV}$ using the expected signal strength and the expected Higgs boson transverse momentum. The LHC bounds have been estimated using various Higgs boson production modes and decay channels. As it can be seen, while in this work only one Higgs production mode and decay has been considered, more sensitivity is achievable on the coefficients $\bar{c}_{W}$

TABLE II. The expected bounds at $95 \% \mathrm{CL}$ on the Wilson coefficients from the LHC [42] at the center-of-mass energy of $14 \mathrm{TeV}$ with $300 \mathrm{fb}^{-1}$ and $3000 \mathrm{fb}^{-1}$ as well the limits obtained form the current analysis in the electron-positron collisions at the center-of-mass energies of $350 \mathrm{GeV}$ and $500 \mathrm{GeV}$ considering only one coefficient in the fit.

\begin{tabular}{llllll}
\hline \hline & LHC-300 & LHC-3000 & $e^{-} e^{+}-350-300$ & $e^{-} e^{+}-350-3000$ & $e^{-} e^{+}-500-300$ \\
\hline $\bar{c}_{W}\left[\times 10^{3}\right]$ & {$[-8.0,8.0]$} & {$[-4.0,4.0]$} & {$[-4.80,3.79]$} & {$[-1.37,1.27]$} & {$[-3.24,2.31]$} \\
$\bar{c}_{H}\left[\times 10^{3}\right]$ & {$[<-50,>50]$} & {$[-44,35]$} & {$[-118.43,129.85]$} & {$[-39.40,40.70]$} & {$[-117.58,145.86]$} \\
$\bar{c}_{H W}\left[\times 10^{3}\right]$ & {$[-7.0,10.0]$} & {$[-4.0,4.0]$} & {$[-6.19,5.52]$} & {$[-1.87,1.80]$} & {$[-3.65,3.03]$} \\
$\bar{c}_{\gamma}\left[\times 10^{3}\right]$ & {$[-1.9,2.2]$} & {$[-0.6,0.7]$} & {$[-61.09,19.78]$} & {$[-19.09,6.25]$} & {$[-43.09,19.64]$} \\
$\bar{c}_{H B}\left[\times 10^{3}\right]$ & {$[-8.0,11.0]$} & {$[-4.0,4.0]$} & {$[-51.35,19.51]$} & {$[-17.20,6.61]$} & {$[-24.70,9.96]$} \\
\hline \hline
\end{tabular}


and $\bar{c}_{H W}$ at the electron-positron colliders with respect to the LHC. In this work, the sensitivity to the dimension-six coefficients is obtained by considering only the Higgs boson decay into a $b \bar{b}$ pair. Including the other Higgs boson decay channels such as $H \rightarrow \gamma \gamma, H \rightarrow W W^{*}, H \rightarrow Z Z^{*}$, and $H \rightarrow \tau \tau$ provides significantly improved sensitivity to dimension-six coefficients. The hadronic and invisible decays of the $Z$ boson as well as using the $W W$-fusion Higgs production channels would be significantly useful to improve the exclusion ranges at the electron-positron colliders.

It is notable that the next-to-leading order corrections [88-90] to the production cross section of $H+Z$ production could modify the shape of the $\cos (\ell, b)$, which needs to be considered in obtaining the sensitivity. To consider such effects, an overall large uncertainty of $10 \%$ in each bin of $\cos (\ell, b)$ distribution is taken into account and the bounds are computed again. For example, the constraints on $\bar{c}_{W}$ and $\bar{c}_{H W}$ at the center-of-mass energy of $350 \mathrm{GeV}$ with an integrated luminosity of $3000 \mathrm{fb}^{-1}$ are as follows: $-0.00144<\bar{c}_{W}<0.00133$ and $-0.00196<\bar{c}_{H W}<0.00189$. Therefore, including a $10 \%$ conservative uncertainty would not weaken the limits significantly.

The above bounds can be used to constrain the parameters of a few explicit models beyond the SM that at the low energy limit reduce to the effective Lagrangian introduced in Eq. (2). In theories with strongly interacting Higgs bosons, the Wilson coefficients are at the order of $[73,75]$

$$
\begin{aligned}
& \bar{c}_{W} \sim O\left(\frac{m_{W}}{M}\right)^{2}, \quad \bar{c}_{H} \sim O\left(\frac{g^{\star} v}{M}\right)^{2}, \\
& \bar{c}_{\gamma} \sim O\left(\frac{m_{W}}{4 \pi}\right)^{2} \times\left(\frac{y_{t}}{M}\right)^{2}, \quad \bar{c}_{H W} \sim O\left(\frac{m_{W}}{4 \pi}\right)^{2} \times\left(\frac{g^{\star}}{M}\right)^{2},
\end{aligned}
$$

where the strength of the Higgs boson coupling to a new physics state is denoted by $g^{\star}$ and $M$ is an overall mass scale of the new possible physical state at which the effective Lagrangian is expected to be matched with the explicit models. As an example, translation of our constraint on $\bar{c}_{W}$ leads to a lower limit of $2.3 \mathrm{TeV}$ on the scale $M$.

\section{SUMMARY AND CONCLUSIONS}

Hints for physics beyond the SM are expected to be found in the Higgs boson sector, which in general could lead to deviations in the Higgs boson couplings with respect to the SM predictions. As a result, indirect searches for new physics via the Higgs boson require precise measurement of the Higgs boson properties that could be performed by future electron-positron colliders.

At the electron-positron colliders with the center-of-mass energy above the $m_{Z}+m_{H}$ threshold, a large number of Higgs bosons could be produced in association with $Z$ bosons. With the clean environment in the $e^{-} e^{+}$colliders, the $H+Z$ events could be tagged easily through the leptonic $Z$ decays and Higgs boson decays into $b \bar{b}$ pairs. The expected very good resolution for lepton and jet momenta measurements and identifications provides the possibility to characterize this final state efficiently. Therefore, a very precise measurement of the total and differential cross section of $H+Z$ can be performed at the future electron-positron colliders. In this work, by performing a comprehensive analysis including the main sources of background processes and response of the detector, we find the potential of a future electron-positron collider to search for new physics originating from a complete set of effective dimension-six operators that can contribute to Higgs boson production associated with a $Z$ boson. We perform an analysis on the differential cross section of the cosine of the angle between the most energetic charged lepton from $Z$ boson decay and the most energetic b-jet from the Higgs boson decay to find the sensitivity of the $e^{-}+e^{+} \rightarrow H+Z$ process to the dimension-six operators. The analysis is done at the center-of-mass energies of 350 and $500 \mathrm{GeV}$ with an ILD-like detector considering the integrated luminosities of $300 \mathrm{fb}^{-1}$ and $3 \mathrm{ab}^{-1}$. It is found that the $e^{-}+$ $e^{+} \rightarrow H+Z$ process has a great sensitivity to dimensionsix operators induced at tree level. We show that highluminosity runs of the future electron-positron colliders would be able to improve the sensitivity of the highluminosity LHC to new physics via the Higgs boson.

\section{ACKNOWLEDGMENTS}

The authors are especially grateful to Sara Khatibi for fruitful discussions. The authors thank the School of Particles and Accelerators, Institute for Research in Fundamental Sciences (IPM) for financial support of this project. H. K. is also thankful the University of Science and Technology of Mazandaran for financial support provided for this research.

\section{APPENDIX: CUT FLOW TABLE FOR THE CENTER-OF-MASS ENERGY OF 350 GeV}

Table III presents the expected cross sections after different combinations of cuts for signal and SM background processes. The numbers are given in units of $\mathrm{fb}$. The signal cross sections correspond to particular values of $\bar{c}_{H}=0.1$ and $\bar{c}_{\gamma}=0.1$. The center-of-mass energy of the collision is assumed to be $\sqrt{s}=350 \mathrm{GeV}$. 
TABLE III. Expected cross sections in units of fb after different combinations of cuts for signal and SM background processes. The signal cross sections correspond to particular values of $\bar{c}_{H}=0.1$ and $\bar{c}_{\gamma}=0.1$. The center-of-mass energy of the collision is assumed to be $350 \mathrm{GeV}$. More details of the selection cuts are given in Sec. III.

\begin{tabular}{|c|c|c|c|c|c|c|}
\hline \multirow{2}{*}{$\begin{array}{l}\sqrt{s}=350 \mathrm{GeV} \\
\text { Cuts }\end{array}$} & \multicolumn{2}{|c|}{ Signal } & \multicolumn{4}{|c|}{ Background } \\
\hline & $\bar{c}_{H}$ & $\bar{c}_{\gamma}$ & $\mathrm{SM}(H+Z)$ & $t \bar{t}$ & $Z Z$ & $Z \gamma, \gamma \gamma, W W Z$ \\
\hline Cross sections (in $\mathrm{fb}$ ) & 10.21 & 26.46 & 11.30 & 10.42 & 59.42 & 20.62 \\
\hline (I): $2 \ell,\left|\eta^{\ell}\right|<2.5, p_{T}^{\ell}>10$ & 7.33 & 18.63 & 8.10 & 6.17 & 40.86 & 7.74 \\
\hline (II): 2 jets, $\left|\eta^{\text {jet }}\right|<2.5, p_{T}^{\text {jet }}>20, \Delta R_{\ell \text {,jet }} \geq 0.5$ & 5.09 & 12.99 & 5.61 & 4.58 & 24.44 & 5.76 \\
\hline (III): $2 b$-jets & 2.00 & 5.10 & 2.21 & 1.73 & 1.87 & 2.30 \\
\hline (IV): $p_{T}^{\ell^{+} \ell^{-}}>100$ & 1.55 & 3.64 & 1.71 & 0.08 & 0.81 & 0.46 \\
\hline (V): $90<m_{b \bar{b}}<160,75<m_{\ell^{+} \ell^{-}}<105$ & 1.284 & 2.997 & 1.410 & 0.003 & 0.122 & 0.016 \\
\hline
\end{tabular}

[1] G. Aad et al. (ATLAS Collaboration), Phys. Lett. B 716, 1 (2012).

[2] S. Chatrchyan et al. (CMS Collaboration), Phys. Lett. B 716, 30 (2012).

[3] (The ATLAS and CMS Collaborations), Report No. ATLAS-CONF-2015-044.

[4] K. Hagiwara, R. Szalapski, and D. Zeppenfeld, Phys. Lett. B 318, 155 (1993).

[5] Z. Han and W. Skiba, Phys. Rev. D 71, 075009 (2005).

[6] T. Corbett, O. J. P. Eboli, J. Gonzalez-Fraile, and M. C. Gonzalez-Garcia, Phys. Rev. D 87, 015022 (2013).

[7] B. Dumont, S. Fichet, and G. von Gersdorff, J. High Energy Phys. 07 (2013) 065.

[8] M. Ciuchini, E. Franco, S. Mishima, M. Pierini, L. Reina, and L. Silvestrini, Nucl. Part. Phys. Proc. 273, 2219 (2016).

[9] J. Ellis, V. Sanz, and T. You, J. High Energy Phys. 07 (2014) 036.

[10] A. Falkowski and F. Riva, J. High Energy Phys. 02 (2015) 039.

[11] A. Falkowski, Pramana 87, 39 (2016).

[12] J. Brehmer, A. Freitas, D. Lopez-Val, and T. Plehn, Phys. Rev. D 93, 075014 (2016).

[13] A. Falkowski, B. Fuks, K. Mawatari, K. Mimasu, F. Riva, and V. sanz, Eur. Phys. J. C 75, 583 (2015).

[14] P. Achard et al. (L3 Collaboration), Phys. Lett. B 583, 14 (2004).

[15] P. Achard et al. (L3 Collaboration), Phys. Lett. B 609, 35 (2005).

[16] A. Pomarol and F. Riva, J. High Energy Phys. 01 (2014) 151.

[17] S. Alam, S. Dawson, and R. Szalapski, Phys. Rev. D 57, 1577 (1998).

[18] S. Taheri Monfared, S. Fayazbakhsh, and M. Mohammadi Najafabadi, Phys. Lett. B 762, 301 (2016).

[19] F. Ferreira, B. Fuks, V. Sanz, and D. Sengupta, arXiv: 1612.01808 .

[20] S. Khatibi and M. Mohammadi Najafabadi, Phys. Rev. D 90, 074014 (2014).

[21] H. Hesari, H. Khanpour, and M. Mohammadi Najafabadi, Phys. Rev. D 92, 113012 (2015).
[22] Y. B. Liu and Z. J. Xiao, Phys. Rev. D 94, 054018 (2016).

[23] H. Khanpour, S. Khatibi, M. Khatiri Yanehsari, and M. Mohammadi Najafabadi, arXiv:1408.2090.

[24] J. Elias-Miró, J. R. Espinosa, E. Masso, and A. Pomarol, J. High Energy Phys. 08 (2013) 033.

[25] J. Elias-Miró, C. Grojean, R. S. Gupta, and D. Marzocca, J. High Energy Phys. 05 (2014) 019.

[26] C. Y. Chen, S. Dawson, and C. Zhang, Phys. Rev. D 89, 015016 (2014).

[27] H. Mebane, N. Greiner, C. Zhang, and S. Willenbrock, Phys. Rev. D 88, 015028 (2013).

[28] N. Craig, M. Farina, M. McCullough, and M. Perelstein, J. High Energy Phys. 03 (2015) 146.

[29] G. J. Gounaris and F. M. Renard, Phys. Rev. D 90, 073007 (2014).

[30] W. Kilian, M. Kramer, and P. M. Zerwas, Phys. Lett. B 373, 135 (1996).

[31] M. Beneke, D. Boito, and Y. M. Wang, J. High Energy Phys. 11 (2014) 028.

[32] S. Heinemeyer, W. Hollik, J. Rosiek, and G. Weiglein, Eur. Phys. J. C 19, 535 (2001).

[33] S. Dawson and S. Heinemeyer, Phys. Rev. D 66, 055002 (2002).

[34] K. Mimasu, V. Sanz, and C. Williams, J. High Energy Phys. 08 (2016) 039.

[35] A. Greljo, G. Isidori, J. M. Lindert, and D. Marzocca, Eur. Phys. J. C 76, 158 (2016).

[36] J. Cohen, S. Bar-Shalom, and G. Eilam, Phys. Rev. D 94, 035030 (2016).

[37] S. F. Ge, H. J. He, and R. Q. Xiao, J. High Energy Phys. 10 (2016) 007.

[38] S. F. Ge, H. J. He, and R. Q. Xiao, Int. J. Geom. Methods Mod. Phys. A31, 1644004 (2016).

[39] N. Craig, J. Gu, Z. Liu, and K. Wang, J. High Energy Phys. 03 (2016) 050.

[40] H. Hesari, H. Khanpour, M. Khatiri Yanehsari, and M. Mohammadi Najafabadi, Adv. High Energy Phys. 2014, 476490 (2014).

[41] A. Arbey, S. Fichet, F. Mahmoudi, and G. Moreau, J. High Energy Phys. 11 (2016) 097. 
[42] C. Englert, R. Kogler, H. Schulz, and M. Spannowsky, Eur. Phys. J. C 76, 393 (2016).

[43] L. Linssen, A. Miyamoto, M. Stanitzki, and H. Weerts, Report No. CERN-2012-003.

[44] M. Aicheler et al., Report No. CERN-2012-007.

[45] H. Abramowicz et al. (CLIC Detector and Physics Study Collaboration), arXiv:1307.5288.

[46] K. Fujii et al., arXiv:1506.05992.

[47] T. Behnke et al., arXiv:1306.6329.

[48] T. Barklow, J. Brau, K. Fujii, J. Gao, J. List, N. Walker, and K. Yokoya, arXiv:1506.07830.

[49] D. M. Asner et al., arXiv:1310.0763.

[50] G. Moortgat-Pick et al., Eur. Phys. J. C 75, 371 (2015).

[51] (CEPC-SPPC Study Group), Reports No. IHEP-CEPCDR-2015-01, No. IHEP-TH-2015-01, and No. IHEP-EP2015-01.

[52] (CEPC-SPPC Study Group), Reports No. IHEP-CEPC-DR2015-01 and No. IHEP-AC-2015-01.

[53] M. Bicer et al. (TLEP Design Study Working Group Collaboration), J. High Energy Phys. 01 (2014) 164.

[54] M. Koratzinos, Proc. Sci., EPS-HEP2015 (2015) 518 [arXiv:1511.01021].

[55] D. d'Enterria, arXiv:1602.05043.

[56] J. Ellis and T. You, J. High Energy Phys. 03 (2016) 089.

[57] P. Janot, J. High Energy Phys. 04 (2015) 182.

[58] D. d'Enterria and P. Z. Skands, Reports No. CERN-PH-TH2015-299 and No. CoEPP-MN-15-13.

[59] M. Benedikt, K. Oide, F. Zimmermann, A. Bogomyagkov, E. Levichev, M. Migliorati, and U. Wienands, arXiv: 1508.03363.

[60] M. Koratzinos et al., arXiv:1506.00918.

[61] F. Zimmermann et al., Report No. CERN-ACC-2014-0262.

[62] D. d'Enterria, Frascati Phys. Ser. 61, 17 (2016).

[63] M. Baak et al., arXiv:1310.6708.

[64] J. Fan, M. Reece, and L. T. Wang, J. High Energy Phys. 09 (2015) 196.

[65] J. Fan, M. Reece, and L. T. Wang, J. High Energy Phys. 08 (2015) 152.

[66] G. Brooijmans et al., arXiv:1605.02684.

[67] T. Han, Z. Liu, Z. Qian, and J. Sayre, Phys. Rev. D 91, 113007 (2015).

[68] C. Hartmann, W. Shepherd, and M. Trott, J. High Energy Phys. 03 (2017) 060.
[69] S. Banerjee, T. Mandal, B. Mellado, and B. Mukhopadhyaya, J. High Energy Phys. 09 (2015) 057

[70] G. Amar, S. Banerjee, S. von Buddenbrock, A. S. Cornell, T. Mandal, B. Mellado and B. Mukhopadhyaya, J. High Energy Phys. 02 (2015) 128

[71] A. Alloul, B. Fuks, and V. Sanz, J. High Energy Phys. 04 (2014) 110.

[72] P. Artoisenet et al., J. High Energy Phys. 11 (2013) 043.

[73] G. F. Giudice, C. Grojean, A. Pomarol, and R. Rattazzi, J. High Energy Phys. 06 (2007) 045.

[74] J. Ellis, V. Sanz, and T. You, J. High Energy Phys. 03 (2015) 157.

[75] R. Contino, M. Ghezzi, C. Grojean, M. Muhlleitner, and M. Spira, J. High Energy Phys. 07 (2013) 035.

[76] R. Barbieri, A. Pomarol, R. Rattazzi, and A. Strumia, Nucl. Phys. B703, 127 (2004).

[77] J. Alwall, C. Duhr, B. Fuks, O. Mattelaer, D. G. Öztürk, and C. H. Shen, Comput. Phys. Commun. 197, 312 (2015).

[78] J. Alwall, R. Frederix, S. Frixione, V. Hirschi, F. Maltoni, O. Mattelaer, H.-S. Shao, T. Stelzer, P. Torrielli, and M. Zaro, J. High Energy Phys. 07 (2014) 079.

[79] J. Alwall, M. Herquet, F. Maltoni, O. Mattelaer, and T. Stelzer, J. High Energy Phys. 06 (2011) 128.

[80] A. Alloul, N. D. Christensen, C. Degrande, C. Duhr, and B. Fuks, Comput. Phys. Commun. 185, 2250 (2014).

[81] T. Sjostrand, S. Mrenna, and P. Z. Skands, Comput. Phys. Commun. 178, 852 (2008).

[82] T. Sjostrand, L. Lonnblad, S. Mrenna, and P. Z. Skands, arXiv:hep-ph/0308153.

[83] J. de Favereau, C. Delaere, P. Demin, A. Giammanco, V. Lemaître, A. Mertens, and M. Selvaggi (DELPHES 3 Collaboration), J. High Energy Phys. 02 (2014) 057.

[84] A. Mertens, J. Phys. Conf. Ser. 608, 012045 (2015).

[85] C. Patrignani et al. (Particle Data Group Collaboration), Chin. Phys. C 40, 100001 (2016).

[86] M. Cacciari, G. P. Salam, and G. Soyez, J. High Energy Phys. 04 (2008) 063.

[87] M. Cacciari, G. P. Salam, and G. Soyez, Eur. Phys. J. C 72, 1896 (2012).

[88] J. Fleischer and F. Jegerlehner, Nucl. Phys. B216, 469 (1983).

[89] B. A. Kniehl, Z. Phys. C 55, 605 (1992).

[90] V. Driesen, W. Hollik, and J. Rosiek, Z. Phys. C 71, 259 (1996). 